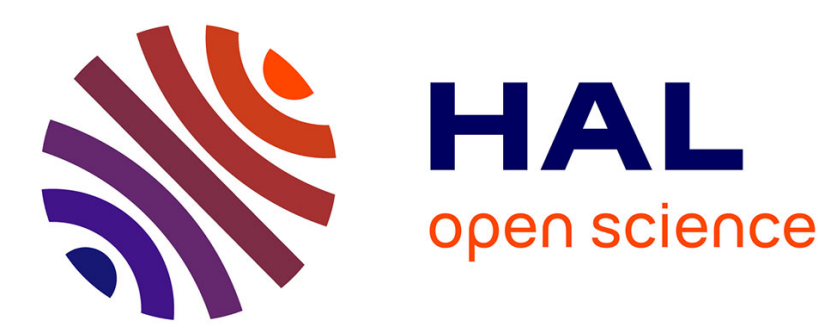

\title{
A Micromechanics-based modification of the Gurson criterion by using Eshelby-like velocity fields
}

\author{
Vincent Monchiet, E. Charkaluk, D. Kondo
}

\section{To cite this version:}

Vincent Monchiet, E. Charkaluk, D. Kondo. A Micromechanics-based modification of the Gurson criterion by using Eshelby-like velocity fields. European Journal of Mechanics - A/Solids, 2011, 30 (6), pp.940-949. 10.1016/j.euromechsol.2011.05.008 . hal-00687856

\section{HAL Id: hal-00687856 https://hal.science/hal-00687856}

Submitted on 18 Apr 2012

HAL is a multi-disciplinary open access archive for the deposit and dissemination of scientific research documents, whether they are published or not. The documents may come from teaching and research institutions in France or abroad, or from public or private research centers.
L'archive ouverte pluridisciplinaire HAL, est destinée au dépôt et à la diffusion de documents scientifiques de niveau recherche, publiés ou non, émanant des établissements d'enseignement et de recherche français ou étrangers, des laboratoires publics ou privés. 


\title{
A Micromechanics-based modification of the Gurson criterion by using Eshelby-like velocity fields.
}

\author{
Vincent MONCHIET ${ }^{\mathrm{a}}$, Eric CHARKALUK ${ }^{\mathrm{b}}$, \\ Djimedo KONDO ${ }^{\mathrm{c}}$
}

${ }^{a}$ Université Paris-Est, Modélisation et Simulation Multi Echelle, MSME UMR 8208 CNRS, 5 boulevard Descartes, 77454 Marne la Vallée, France

${ }^{\mathrm{b}}$ Université de Lille I, Laboratoire de Mécanique de Lille, LML UMR 8107 CNRS, Cité Scientifique, Bd. Paul Langevin, 59655 Villeneuve d'Ascq, France

${ }^{\mathrm{c}}$ Université Pierre et Marie Curie, Institut D'Alembert, UMR 7190 CNRS, 4, Place Jussieu, 75252 Paris Cedex 05, France

\begin{abstract}
In this study, we propose a micromechanics-based modification of the Gurson criterion for porous media subjected to arbitrary loadings. The proposed formulation, derived in the framework of limit analysis, consists in the consideration of Eshelbylike exterior point trial velocity fields for the determination of the macroscopic dissipation. This approach is implemented for perfectly plastic rigid von Mises matrix containing spherical voids. After the minimization procedure required by the use of the Eshelby-like trial velocity fields, we derive a two-field estimate of the macroscopic yield function. It is shown that the obtained closed-form estimate provides a significant modification of the Gurson criterion, particularly in the domain of low stress triaxialities. This estimate is first compared with existing criteria. Moreover, its accuracy is assessed through comparison with results derived from numerical exact two-field criterion and with recently available numerical bounds.
\end{abstract}

Key words: ductile porous materials, Gurson type model, spherical cavities, Eshelby-like trial velocity fields, micromechanics, limit analysis

Email addresses: vincent.monchiet@univ-mlv.fr (Vincent MONCHIET), eric.charkaluk@univ-lille1.fr (Eric CHARKALUK), djimedo.kondo@upmc.fr (Djimedo KONDO). 


\section{Introduction}

During the four past decades, the field of ductile damage has been the subject of important researches in non linear solids mechanics. Among the first to study the mechanical behavior of porous materials, McClintock [16] and Rice and Tracey [27] derived, from a variational procedure, a cavity growth law for spherical and cylindrical voids in a von Mises matrix. These pioneering works were developed in the domain of dilute concentration of voids, which justifies an uncoupling between the plastic behavior of the matrix and the void growth. For finite porosities, Gurson [10] developed a limit analysis approach of a hollow sphere made up of a rigid ideal plastic von Mises material and subjected to an arbitrary loading. In the case of homogeneous strain rate boundary conditions, Gurson derived a macroscopic yield function by considering two velocity fields: the first one corresponds to a homogeneous strain rate, while the second one takes into account the cavity expansion. For the latter, Gurson makes use of the exact solution of the hollow sphere subjected to a hydrostatic external loading. Consequently, the obtained macroscopic yield surface provides the exact solution in this particular loading case. Moreover, it was shown (see for instance [13]) that the Gurson yield surface constitutes an upper bound for porous media, at least for Hashin composite-sphere assemblage. Later, Tvergaard [31] observed that Gurson model is too stiff when compared with finite element unit-cell computations. This observation motivated the heuristic extension of the Gurson model which has been proposed by Tvergaard and Needleman [32]. Such extension, known as the GTN model, introduces three parameters, $q_{1}, q_{2}$ and $q_{3}$, which have to be determined ${ }^{1}$.

Alternatively, making use of variational techniques in non linear homogenization framework, Ponte-Castañeda [24] ${ }^{2}$ obtained rigorous Hashin-Shtrikman type upper bounds for a von Mises plastic materials containing spherical voids. An important observation is that the Gurson model violates the non linear homogenization-based upper bound for low values of the stress triaxiality $T=\Sigma_{m} / \Sigma_{e q}$. However, the Gurson model predicts values more realistic than the Hashin-Shtrikman bound which yields unreasonably high values. Note that Danas and Ponte-Castañeda [2] recently derived accurate yield surfaces for porous materials by using a second order homogenization method. A notable advantage of this second order approach is to exhibits an effect of the third invariant for isotropic microstructures. Note also that this effect has also be investigated in recent numerical studies by Thore et al. (2011). However, since one major advantage of the limit analysis approach over the latter is that it

1 For completeness, mention must be made of [14] and of several extensions of the Gurson model accounting for voids shape effects (see for instance [7], [8], [9], [6], [22], [1], [19])

2 A similar derivation has been done by Suquet [29] for cylindrical voids. 
provides closed-form expression of the yield function, it would be interesting to improve the predictions of the original Gurson model. A possible way to do this consists to consider refined trial velocity fields still in the limit analysis framework. For instance, this was done by $[5,6]$ who considered the exact solution of the elastic hollow sphere subjected to an arbitrary loading. However this criterion still violates the Hashin-Shtrikman bounds.

The main objective of the present paper is to develop a limit analysis approach based on Eshelby-like velocity fields and to derive new expression of the yield function in the case of spherical voids. The paper is organized as follows. In section 2, we briefly recall the Gurson approach and state the trial velocity fields used in order to improve it (see subsection 2.2). Then, we discuss the question of boundary conditions associated to the consideration of the Eshelby-type velocity field and point out the requirement of a minimization procedure to derive the macroscopic yield surface. Section 3 is devoted to the determination of a closed-form expression of the macroscopic yield function. To this end, some approximations are introduced (see subsection 3.1). The minimization procedure is described in subsection 3.2. In order to underline the improvement of the new obtained criterion, some comparisons with other criteria (Gurson [10] and Hashin-Shtrikman [24]) are provided in section 4.

\section{Limit analysis approach and Eshelby-based velocity field}

\subsection{Statement of the Gurson approach}

Since substantial modifications of the Gurson limit analysis will be proposed, we briefly recall here the main steps of the methodology which will be followed. In the following we consider the spherical basis $\left(\underline{e}_{r}, \underline{e}_{\theta}, \underline{e}_{\varphi}\right)$ and the associated coordinates system $(r, \theta, \varphi)$, with $\theta \in[0,2 \pi]$ and $\varphi \in[0, \pi]$. Let us consider a hollow sphere with an external radius $b$ and a void of radius $a$. This hollow sphere is subjected at its outer boundary to a homogeneous strain rate conditions:

$$
\underline{v}(r=b)=D \cdot \underline{x}
$$

The determination of an approximate macroscopic criterion, in the framework of limit analysis requires the choice of the velocity field into the solid matrix. As classically, and in agreement with [10], we adopt the velocity in the following general form: 


$$
\underline{v}=\boldsymbol{A} \cdot \underline{x}+\underline{v}^{E}
$$

in which the term $\boldsymbol{A} . x$ is associated to a uniform strain rate $\boldsymbol{A}$ and $\underline{v}^{E}$ is a heterogeneous field which corresponds to the expansion of the cavity. The matrix incompressibility implies that $\operatorname{tr}(\boldsymbol{A})=0$ and $\operatorname{div}\left(\underline{v}^{E}\right)=0$. For $\underline{v}^{E}$, the choice made by Gurson corresponds to the solution of the plastic hollow sphere subjected to an hydrostatic loading; this field takes the form:

$$
\underline{v}^{E}=\frac{C}{r^{2}} \underline{e}_{r}
$$

in which $C$ is a constant. The consideration of the boundary conditions leads to:

$$
\underline{v}=\overline{\boldsymbol{D}} \cdot \underline{x}+D_{m} \frac{b^{3}}{r^{2}} \underline{e}_{r}
$$

where $D_{m}=\frac{1}{3} \operatorname{tr}(\boldsymbol{D})$ and $\overline{\boldsymbol{D}}$ represents the deviatoric part of $\boldsymbol{D}$.

It is assumed that the matrix material obeys to a von Mises criterion, with $\sigma_{0}$ the uniaxial tensile yield stress (material constant). Accordingly, the macroscopic plastic dissipation $\Pi(\boldsymbol{D})$ is defined as:

$$
\Pi(\boldsymbol{D})=\frac{\sigma_{0}}{|\Omega|} \int_{\Omega-\omega} d_{e q} d V
$$

where $d_{e q}$ is the equivalent strain rate, $d_{e q}=\sqrt{\frac{2}{3} \overline{\boldsymbol{d}}: \overline{\boldsymbol{d}}}$. Tensor $\overline{\boldsymbol{d}}$ is the deviatoric part of the strain rate tensor $\boldsymbol{d}$ deduced from the velocity field (4). The following inequality then holds for all macroscopic stresses, $\boldsymbol{\Sigma}$ and strain rate, $\boldsymbol{D}$ (Hill-Mandel lemma):

$$
\Sigma: D \leq \Pi(D)
$$

As a consequence, the macroscopic yield locus of the plastic porous medium is given by:

$$
\boldsymbol{\Sigma}=\frac{\partial \Pi}{\partial \boldsymbol{D}}
$$

In the case of von Mises matrix, the computation performed by Gurson, using (4), leads to:

$$
\frac{\Sigma_{e q}^{2}}{\sigma_{0}^{2}}+2 f \cosh \left(\frac{3}{2} \frac{\Sigma_{m}}{\sigma_{0}}\right)-1-f^{2} \leq 0
$$


where $\Sigma_{e q}$ is the macroscopic von Mises equivalent stress, $\Sigma_{e q}=\sqrt{\frac{3}{2} \bar{\Sigma}: \bar{\Sigma}}$, $\Sigma_{m}=\frac{1}{3} \operatorname{tr}(\boldsymbol{\Sigma})$ and $f$ is the material porosity.

As indicated before and proposed by [5], an improvement of the above yield function can be expected by investigating other velocity fields than the one used by Gurson.

\subsection{Eshelby-based velocity field}

Our proposal is to adopt, for $\underline{v}^{E}$, a field inspired from the solution of the Eshelby inclusion problem [3], [4]. To this end, consider an infinite linear viscous medium in which a finite subdomain $\omega$ has a spherical shape with radius $a$. This subdomain is subjected to a uniform eigenstrain rate $\boldsymbol{d}^{*}$. From Eshelby's well-known results, the strain rate field is homogeneous inside the inclusion, while for an exterior point (outside the inclusion), it is heterogeneous. In the particular case of a spherical inclusion, the exterior point solution reads [4] (see also [21] or [12]):

$$
\underline{v}^{E}=\frac{a^{5}}{5 r^{4}}\left[5 d_{m}^{*} \mathbf{1}+2 \overline{\boldsymbol{d}}^{*}\right] \cdot \underline{e}_{r}+\frac{a^{3}}{r^{2}}\left[1-\frac{a^{2}}{r^{2}}\right] d_{r r}^{*} \underline{e}_{r}
$$

in which $d_{m}^{*}=\frac{1}{3} \operatorname{tr}\left(\boldsymbol{d}^{*}\right)$ and $\overline{\boldsymbol{d}}^{*}$ represents the deviatoric part of $\boldsymbol{d}^{*}$.

In spherical coordinates, $\underline{v}^{E}$ is expressed:

$$
v_{r}^{E}=\frac{a^{3}}{r^{2}}\left[\left(1-\frac{3 a^{2}}{5 r^{2}}\right) d_{r r}^{*}+\frac{3 a^{2}}{5 r^{2}} d_{m}^{*}\right] ; \quad v_{\theta}^{E}=\frac{2 a^{5}}{5 r^{4}} d_{r \theta}^{*} ; \quad v_{\varphi}^{E}=\frac{2 a^{5}}{5 r^{4}} d_{r \varphi}^{*}
$$

where $d_{r r}^{*}, d_{r \theta}^{*}$ and $d_{r \varphi}^{*}$ are given by:

$$
\begin{aligned}
d_{r r}^{*}= & \boldsymbol{d}^{*}:\left(\underline{e}_{r} \otimes \underline{e}_{r}\right)=d_{m}^{*}+\left(1-3 \cos ^{2}(\varphi)\right) d_{2}^{*}-\sin ^{2}(\varphi) \cos (2 \theta) d_{3}^{*} \\
& +\sin ^{2}(\varphi) \sin (2 \theta) d_{4}^{*}+\sin (2 \varphi) \cos (\theta) d_{5}^{*}+\sin (2 \varphi) \sin (\theta) d_{6}^{*} \\
d_{r \varphi}^{*}= & \overline{\boldsymbol{d}}^{*}:\left(\underline{e}_{r} \otimes \underline{e}_{\varphi}\right)=\frac{3}{2} \sin (2 \varphi) d_{2}^{*}-\frac{1}{2} \sin (2 \varphi) \cos (2 \theta) d_{3}^{*} \\
& +\frac{1}{2} \sin (2 \varphi) \sin (2 \theta) d_{4}^{*}+\cos (2 \varphi) \cos (\theta) d_{5}^{*}+\cos (2 \varphi) \sin (\theta) d_{6}^{*} \\
d_{r \theta}^{*}= & \overline{\boldsymbol{d}}^{*}:\left(\underline{e}_{r} \otimes \underline{e}_{\theta}\right)=\sin (\varphi) \sin (2 \theta) d_{3}^{*}+\sin (\varphi) \cos (2 \theta) d_{4}^{*} \\
& -\cos (\varphi) \sin (\theta) d_{5}^{*}+\cos (\varphi) \cos (\theta) d_{6}^{*}
\end{aligned}
$$


with:

$$
\begin{aligned}
& d_{2}^{*}=\frac{1}{3}\left[\frac{d_{11}^{*}+d_{22}^{*}}{2}-d_{33}^{*}\right] ; \quad d_{3}^{*}=\frac{d_{22}^{*}-d_{11}^{*}}{2} \\
& d_{4}^{*}=d_{12}^{*} ; \quad d_{5}^{*}=d_{13}^{*} ; \quad d_{6}^{*}=d_{23}^{*}
\end{aligned}
$$

It must be emphasized that the Eshelby-based velocity field allows to recover the one considered by Rice and Tracey [27] and by Gurson [10] as a particular case associated to $\overline{\boldsymbol{d}}^{*}=0$ and $d_{m}^{*}=C / a^{2}$ in (9). This corresponds to the case of an inclusion subjected to uniform dilatational eigenstrain rate. Note that the deviatoric part of $\boldsymbol{d}^{*}$ induces the inclusion shape change. Moreover, the resulting field, (2), corresponding to the Eshelby-like velocity field introduces 11 unknown kinematic parameters (the components of $\boldsymbol{d}^{*}$ and $\boldsymbol{A}$ ). The strain rate field $\boldsymbol{d}$ derived from the velocity field (2) with (9) reads:

$$
\boldsymbol{d}=\boldsymbol{A}+\boldsymbol{d}^{E}
$$

with $\boldsymbol{d}^{E}=\nabla_{s} \underline{v}^{E}$, the symmetric part of the velocity gradient associated to $\underline{v}^{E}$. The components of the $\boldsymbol{d}^{E}$ are given by:

$$
\begin{aligned}
& d_{r r}^{E}=-\frac{2 a^{3}}{r^{3}} d_{r r}^{*}+\frac{4}{5} \frac{a^{5}}{r^{5}}\left[2 d_{r r}^{*}-d_{\theta \theta}^{*}-d_{\varphi \varphi}^{*}\right] \\
& d_{\varphi \varphi}^{E}=\frac{a^{3}}{r^{3}} d_{r r}^{*}+\frac{1}{5} \frac{a^{5}}{r^{5}}\left[-4 d_{r r}^{*}+d_{\theta \theta}^{*}+3 * d_{\varphi \varphi}^{*}\right] \\
& d_{\theta \theta}^{E}=\frac{a^{3}}{r^{3}} d_{r r}^{*}+\frac{1}{5} \frac{a^{5}}{r^{5}}\left[-4 d_{r r}^{*}+d_{\theta \theta}^{*}+3 d_{\varphi \varphi}^{*}\right] \\
& d_{r \varphi}^{E}=\frac{a^{3}}{r^{3}}\left[1-\frac{8}{5} \frac{a^{2}}{r^{2}}\right] d_{r \varphi}^{*} ; \quad d_{r \theta}^{E}=\frac{a^{3}}{r^{3}}\left[1-\frac{8}{5} \frac{a^{2}}{r^{2}}\right] d_{r \theta}^{*} ; \quad d_{\varphi \theta}^{E}=\frac{2}{5} \frac{a^{5}}{r^{5}} d_{\theta \varphi}^{*}
\end{aligned}
$$

and can be put on the form:

$$
\boldsymbol{d}^{E}=\mathbb{D}: \boldsymbol{d}^{*}
$$

where $\mathbb{D}$ is a fourth order tensor, which has the minor symmetries $D_{i j k l}=$ $D_{j i k l}=D_{i j l k}$ but not the major symmetry $D_{i j k l} \neq D_{k l i j}$. Components of tensor $\mathbb{D}$ are given by: 


$$
\begin{aligned}
& D_{r r r r}=-2 D_{\theta \theta r r}=-2 D_{\varphi \varphi r r}=-2 \frac{a^{3}}{r^{3}}\left[1-\frac{4}{5} \frac{a^{2}}{r^{2}}\right] \\
& D_{\theta \theta \theta \theta}=D_{\varphi \varphi \varphi \varphi}=\frac{3}{5} \frac{a^{5}}{r^{5}} \quad D_{r r \theta \theta}=D_{r r \varphi \varphi}=-\frac{4}{5} \frac{a^{5}}{r^{5}} \\
& D_{\theta \theta \varphi \varphi}=D_{\varphi \varphi \theta \theta}=D_{\theta \varphi \theta \varphi}=\frac{1}{5} \frac{a^{5}}{r^{5}} \quad D_{r \theta r \theta}=D_{r \varphi r \varphi}=\frac{1}{2} \frac{a^{3}}{r^{3}}\left[1-\frac{8}{5} \frac{a^{2}}{r^{2}}\right]
\end{aligned}
$$

Note that the components of the fourth order tensor $\mathbb{D}$ are such that $D_{\text {rrrr }}+$ $D_{\theta \theta r r}+D_{\varphi \varphi r r}=0$, which corresponds to the matrix incompressibility.

\subsection{Analysis of the associated boundary conditions}

The verification of homogeneous strain rate boundary conditions (1), for the velocity field (2) with (9), implies:

$$
\begin{aligned}
& A_{r r}+f\left[\left(1-\frac{3 f^{2 / 3}}{5}\right) d_{r r}^{*}+\frac{3 f^{2 / 3}}{5} d_{m}^{*}\right]=D_{r r} \\
& A_{r \theta}+\frac{2 f^{5 / 3}}{5} d_{r \theta}^{*}=D_{r \theta} ; \quad A_{r \varphi}+\frac{2 f^{5 / 3}}{5} d_{r \varphi}^{*}=D_{r \varphi}
\end{aligned}
$$

Introducing the deviatoric and mean part of $\boldsymbol{A}, \boldsymbol{d}^{*}$ and $\boldsymbol{D}$ into (17) (i.e. $\left.\boldsymbol{A}=\overline{\boldsymbol{A}}, \boldsymbol{d}^{*}=d_{m}^{*} \mathbf{1}+\overline{\boldsymbol{d}}^{*}, \boldsymbol{D}=D_{m} \mathbf{1}+\overline{\boldsymbol{D}}\right)$ gives:

$$
\begin{aligned}
& f d_{m}^{*}=D_{m} \\
& A_{r r}+f\left(1-\frac{3 f^{2 / 3}}{5}\right) \bar{d}_{r r}^{*}=\bar{D}_{r r} \\
& A_{r \theta}+\frac{2 f^{5 / 3}}{5} \bar{d}_{r \theta}^{*}=\bar{D}_{r \theta} ; \quad A_{r \varphi}+\frac{2 f^{5 / 3}}{5} \bar{d}_{r \varphi}^{*}=\bar{D}_{r \varphi}
\end{aligned}
$$

Solution of (18) is:

$$
d_{m}^{*}=\frac{D_{m}}{f} ; \quad \boldsymbol{A}=\overline{\boldsymbol{D}} ; \quad \overline{\boldsymbol{d}}^{*}=0
$$

It appears that the considered class of Eshelby-based velocity fields ((2) with (9)) complies with the homogeneous strain rate boundary conditions if and only if $\overline{\boldsymbol{d}}^{*}=0$. This is equivalent to $d_{r r}^{*}=$ constant. The velocity field (2) with (9) reduces then to (4) used by Gurson. It follows that all supplementary velocity fields (associated to $\overline{\boldsymbol{d}}^{*} \neq 0$ ) cannot comply with the macroscopic strain rate tensor $\boldsymbol{D}$. In fact, the consideration of the Eshelby-based velocity 
field can be rigorously justified in the context of limit analysis only for homogeneous stress boundary conditions, $\boldsymbol{\sigma} \cdot \underline{n}=\boldsymbol{\Sigma} . \underline{n}$. Then, and in agreement with Hill-Mandel lemma, the macroscopic strain rate $\boldsymbol{D}$ is related to the local strain rate, $\boldsymbol{d}$, by the average rule:

$$
\boldsymbol{D}=\frac{1}{|\Omega|} \int_{\Omega} \boldsymbol{d} d V
$$

Note that, the use of the kinematic approach (developed in section 3) leads to an upper bound of the macroscopic yield surface in the context of the homogeneous stress boundary conditions.

Introducing now (13) with (15) into (20) leads to:

$$
\boldsymbol{D}=\frac{1}{|\Omega|} \int_{\Omega} \boldsymbol{d} d V=\boldsymbol{A}+f\left[d_{m}^{*}+\frac{2}{5} \overline{\boldsymbol{d}}^{*}\right]
$$

which links the macroscopic strain rate $\boldsymbol{D}$ to $\boldsymbol{A}$ and $\boldsymbol{d}^{*}$. Consideration of the deviatoric and the mean part of $\boldsymbol{D}$ gives:

$$
\left\{\begin{array}{l}
\overline{\boldsymbol{D}}=\boldsymbol{A}+\frac{2 f}{5} \overline{\boldsymbol{d}}^{*} \\
D_{m}=f d_{m}^{*}
\end{array}\right.
$$

In these relations, one can observe that the mean part of the tensor $\boldsymbol{d}^{*}$ is known and explicitly given in terms of the macroscopic strain rate, $D_{m}$. Moreover $(22)$ provides 6 scalar relations for the 11 unknown parameters (the components of $\boldsymbol{A}$ and $\boldsymbol{d}^{*}$. It still remains 5 parameters, namely the components of $\overline{\boldsymbol{d}}^{*}$, whose determination will imply a minimization procedure.

\section{The two-field estimate of the macroscopic yield criterion}

\subsection{The approximate macroscopic dissipation}

This section is devoted to the determination of an approximate criterion by considering the Eshelby-based trial velocity field. Although the general methodology has been depicted in the section 2.1, it must be emphasized that our approach presents some substantial differences with the Gurson one. Indeed, as mentioned before, the macroscopic plastic dissipation will be obtained after a minimization procedure on the remaining unknown parameters: 


$$
\Pi(\boldsymbol{D})=\min _{\overline{\boldsymbol{d}}^{*}}\left[\Gamma\left(\boldsymbol{D}, \overline{\boldsymbol{d}}^{*}\right)\right]
$$

with:

$$
\Gamma\left(\boldsymbol{D}, \overline{\boldsymbol{d}}^{*}\right)=\frac{\sigma_{0}}{|\Omega|} \int_{\Omega-\omega} d_{e q} d V
$$

in which

$$
d_{e q}^{2}=A_{e q}^{2}+\frac{4}{3} \boldsymbol{A}: \mathbb{D}: \boldsymbol{d}^{*}+\frac{2}{3} \boldsymbol{d}^{*}: \mathbb{D}^{T}: \mathbb{D}: \boldsymbol{d}^{*}
$$

We now propose to derive an explicit expression of $\Gamma(\boldsymbol{D}, \overline{\boldsymbol{d}})$ which requires the integration of the equivalent strain rate $d_{e q}$ as given by (25). Let us introduce the first approximation used to perform the integration of the local dissipation:

$\mathcal{A} 1$ : The mean value of $d_{e q}$ over the unit sphere, i.e. $\left\langle d_{e q}>_{S(r)}\right.$, is replaced by $\sqrt{<d_{e q}^{2}>_{S(r)}}$, such as:

$$
<d_{e q}^{2}>_{S(r)}=A_{e q}^{2}+\frac{4}{3} \boldsymbol{A}:<\mathbb{D}>_{S(r)}: \boldsymbol{d}^{*}+\frac{2}{3} \boldsymbol{d}^{*}:<\mathbb{D}^{T}: \mathbb{D}>_{S(r)}: \boldsymbol{d}^{*}
$$

Note that this approximation has been used by [10] and has the advantage to preserve the upper bound character of the derived macroscopic yield function. As the mean value of $\mathbb{D}$ over the unit sphere is zero, $<\mathbb{D}>_{S(r)}=0$, the crossed term is null. Concerning the term $\left\langle\mathbb{D}^{T}: \mathbb{D}>_{S(r)}\right.$, its computation gives:

$$
<\mathbb{D}^{T}: \mathbb{D}>_{S(r)}=\left\{2 \mathbb{J}+\frac{6}{25} G(u) \mathbb{K}\right\} u^{2}
$$

with $\mathbb{J}=\frac{1}{3} \mathbf{1} \otimes \mathbf{1}, \mathbf{1}$ being the second order unit tensor. Tensor $\mathbb{K}$ is defined by $\mathbb{K}=\mathbb{I}-\mathbb{J}$, with $\mathbb{I}$ the fourth order symmetric identity tensor. $G(u)$ is a function of variable $u=a^{3} / r^{3}$, defined by:

$$
G(u)=\frac{1}{3}\left(15-40 u^{2 / 3}+28 u^{4 / 3}\right)
$$

To summarize, $<d_{e q}^{2}>_{S(r)}$ is expressed as:

$$
<d_{e q}^{2}>_{S(r)}=A_{e q}^{2}+\left[4 d_{m}^{* 2}+\frac{6 G(u)}{25} d_{e q}^{* 2}\right] u^{2}
$$


With approximation $\mathcal{A} 1, \Gamma(\boldsymbol{D}, \overline{\boldsymbol{d}})$ reads:

$$
\Gamma(\boldsymbol{D}, \overline{\boldsymbol{d}})=\sigma_{0} f \int_{u=f}^{u=1} \sqrt{A_{e q}^{2}+\left(4 d_{m}^{*}+\frac{6 G(u)}{25} d_{e q}^{*}\right) u^{2}} \frac{d u}{u^{2}}
$$

We aim now at deriving a closed-form expression of $\Gamma(\boldsymbol{D}, \overline{\boldsymbol{d}})$. Due to the presence of $G(u)$ in (30), a second approximation is needed, the objective being to put $\Gamma(\boldsymbol{D}, \overline{\boldsymbol{d}})$ in the form $\sigma_{0} f \int_{f}^{1} \sqrt{A^{2}+B^{2} u^{2}} \frac{d u}{u^{2}}$ (which can be easily computed) where $A$ and $B$ are two constants. This second approximation is expressed as:

$\mathcal{A} 2$ : function $G(u)$ is replaced by its mean value, denoted thereafter $g(f)$, over the interval $\left[u_{1}, u_{2}\right]=[1, f]$

The computation of $g(f)$ leads to:

$$
g(f)=\frac{1}{1-f} \int_{f}^{1} G(u) d u=1-4 f \frac{\left(1-f^{2 / 3}\right)^{2}}{1-f}
$$

from which:

$$
\begin{aligned}
\Gamma\left(\boldsymbol{D}, \overline{\boldsymbol{d}}^{*}\right)= & \sigma_{0} f \int_{f}^{1}\left\{A_{e q}^{2}+B^{2} u^{2}\right\}^{1 / 2} \frac{d u}{u^{2}} \\
& =\sigma_{0} f\left[B \operatorname{arcsinh}\left\{\frac{u B}{A_{e q}}\right\}-\frac{\sqrt{A_{e q}^{2}+u^{2} B^{2}}}{u}\right]_{f}^{1}
\end{aligned}
$$

with

$$
B^{2}=4 d_{m}^{* 2}+\frac{6 g(f)}{25} d_{e q}^{* 2}=\frac{4}{f^{2}} D_{m}^{2}+\frac{6 g(f)}{25} d_{e q}^{* 2}
$$

Note that, in this form, $\Gamma\left(\boldsymbol{D}, \overline{\boldsymbol{d}}^{*}\right)$ is equivalent to the one obtained by Gurson in the case $d_{e q}^{*}=0$ and $A_{e q}=D_{e q}$.

It is important to emphasize that, since any inequality can be established, $\mathcal{A} 2$ introduces an "uncontrolled" approximation in the computation of $\Gamma\left(\boldsymbol{D}, \overline{\boldsymbol{d}}^{*}\right)$. Consequently, at the difference of Gurson result, the upper bound character of the approximate criterion which will be established in the following is not ensured. Note however that such upper bound can be numerically determined starting from (34) in which $\Gamma\left(\boldsymbol{D}, \overline{\boldsymbol{d}}^{*}\right)$ is obtained from a numerical integration. 
The main objective of the present section is to determine an expression of the macroscopic yield function from the procedure depicted in section (3.1). This requires first the determination of the unknown parameters $\overline{\boldsymbol{d}}^{*}$ from the minimization of $\Gamma\left(\boldsymbol{D}, \overline{\boldsymbol{d}}^{*}\right)$. Once this minimization is performed, it still remains to determine the macroscopic yield surface from (7). However, it was possible to perform these two steps at the same time as ${ }^{3}$ :

$$
\boldsymbol{\Sigma}=\frac{\partial \Gamma\left(\boldsymbol{D}, \overline{\boldsymbol{d}}^{*}\right)}{\partial \boldsymbol{D}} \text { with: } \frac{\partial \Gamma\left(\boldsymbol{D}, \overline{\boldsymbol{d}}^{*}\right)}{\partial \overline{\boldsymbol{d}}^{*}}=0
$$

A complete resolution of this non linear system can be done by introducing into (34) the following change of variables: $\Gamma\left(\boldsymbol{D}, \overline{\boldsymbol{d}}^{*}\right)=\Gamma\left(A_{e q}, B\right)$. In one hand, the macroscopic stress tensor $\boldsymbol{\Sigma}$ is given by:

$$
\boldsymbol{\Sigma}=\frac{\partial \Gamma}{\partial A_{e q}} \frac{\partial A_{e q}}{\partial \boldsymbol{D}}+\frac{\partial \Gamma}{\partial B} \frac{\partial B}{\partial \boldsymbol{D}}
$$

In another hand, in (34), the second relation reads:

$$
\frac{\partial \Gamma}{\partial A_{e q}} \frac{\partial A_{e q}}{\partial \overline{\boldsymbol{d}}^{*}}+\frac{\partial \Gamma}{\partial B} \frac{\partial B}{\partial \overline{\boldsymbol{d}}^{*}}=0
$$

Derivatives of $A_{e q}$ and $B$ according to $\boldsymbol{D}$ and $\overline{\boldsymbol{d}}^{*}$ provide:

$$
\begin{array}{ll}
\frac{\partial A_{e q}}{\partial \boldsymbol{D}}=\frac{2}{3} \frac{\boldsymbol{A}}{A_{e q}} ; & \frac{\partial A_{e q}}{\partial \overline{\boldsymbol{d}}^{*}}=-f \frac{4}{15} \frac{\boldsymbol{A}}{A_{e q}} \\
\frac{\partial B}{\partial \boldsymbol{D}}=\frac{4 D_{m}}{3 f^{2} B} \mathbf{1} ; & \frac{\partial B}{\partial \overline{\boldsymbol{d}}^{*}}=\frac{4 g(f)}{25 B} \overline{\boldsymbol{d}}^{*}
\end{array}
$$

Introducing expressions in (37) into (35) and (36) gives the following expressions for the mean and equivalent macroscopic stress, as well as the minimum condition:

$\overline{3 \text { Note }}$ that the computation of $\overline{\boldsymbol{d}}^{*}$ has been done and the result is reported in appendix $A$. 


$$
\begin{aligned}
\Sigma_{m} & =\frac{4}{3} \frac{\partial \Gamma}{\partial B} \frac{D_{m}}{f^{2} B} \\
\Sigma_{e q} & =\frac{\partial \Gamma}{\partial A_{e q}} \\
\frac{\partial \Gamma}{\partial A_{e q}} & =\frac{3 g(f)}{5 f} \frac{d_{e q}^{*}}{B} \frac{\partial \Gamma}{\partial B}
\end{aligned}
$$

The elimination of $B$ between the first and the last equation in (38) leads to:

$$
\begin{aligned}
& \frac{\partial \Gamma}{\partial A_{e q}}=\Sigma_{e q} \\
& \frac{\partial \Gamma}{\partial B}=f \sqrt{\frac{9}{4} \Sigma_{m}^{2}+\frac{2}{3 g(f)} \Sigma_{e q}^{2}}
\end{aligned}
$$

The computation of $\frac{\partial \Gamma}{\partial A_{e q}}$ and $\frac{\partial \Gamma}{\partial B}$ is performed by considering (32):

$$
\begin{gathered}
\frac{\partial \Gamma}{\partial A_{e q}}=\sigma_{0}\left[\sqrt{1+f^{2} \xi^{2}}-f \sqrt{1+\xi^{2}}\right] \\
\frac{\partial \Gamma}{\partial B}=\sigma_{0} f[\operatorname{arcsinh}(\xi)-\operatorname{arcsinh}(f \xi)]
\end{gathered}
$$

with $\xi=B / A_{e q}, B$ being given by (33).

The macroscopic criterion is obtained by replacing the derivatives of $\Gamma$ in (40) by (39) and eliminating $\xi$ in (40):

$$
F(\boldsymbol{\Sigma}, f)=\frac{\Sigma_{e q}^{2}}{\sigma_{0}^{2}}+2 f \cosh \left(\sqrt{\frac{9}{4} \frac{\Sigma_{m}^{2}}{\sigma_{0}^{2}}+\frac{2}{3 g(f)} \frac{\Sigma_{e q}^{2}}{\sigma_{0}^{2}}}\right)-1-f^{2} \leq 0
$$

which is the most important practical result of the present paper. In the case of low values of the porosity, one can consider for simplicity that $g(f) \simeq 1$ which leads to:

$$
F(\boldsymbol{\Sigma}, f) \simeq \frac{\Sigma_{e q}^{2}}{\sigma_{0}^{2}}+2 f \cosh \left(\sqrt{\frac{9}{4} \frac{\Sigma_{m}^{2}}{\sigma_{0}^{2}}+\frac{2}{3} \frac{\Sigma_{e q}^{2}}{\sigma_{0}^{2}}}\right)-1-f^{2} \leq 0
$$

Remark: it must be emphasized that the closed form expression of the macroscopic criterion depends on the different approximations considered for the derivation of $\Gamma(\boldsymbol{D}, \overline{\boldsymbol{d}})$. Indeed, consideration of alternative approximation instead of $\mathcal{A} 2$ (see section 3.1) may lead to a criterion different of (41). This has been done and reported in appendix B. 


\section{Evaluation and validation of the established criterion}

We aim now at comparing the new macroscopic yield function (41) with existing criteria. Moreover, the accuracy of this criterion will be evaluated by its comparison with recent numerical bounds.

\subsection{Comparison with existing criteria}

The main difference of the new criterion (41) with (8) lies in the presence of the term introduced by the equivalent stress and the porosity into the hyperbolic cosine. However, the new criterion still preserves, as Gurson's one, the exact solution of the hollow sphere subjected to an hydrostatic loading, $\Sigma_{m}=-\frac{2 \sigma_{0}}{3} \ln (f)$. This is a consequence of the fact that the velocity field considered in the present study contains the one already used by Gurson. Moreover, it is interesting to compare the obtained results to the one deduced from non linear variational homogenization techniques proposed by Ponte Castañeda [24], [25] and Suquet [29]. Indeed, for a representative elementary volume of a porous medium with spherical voids, these authors established a macroscopic elliptic criterion which reads [24]:

$$
\left(1+\frac{2 f}{3}\right) \frac{\Sigma_{e q}^{2}}{\sigma_{0}^{2}}+\frac{9 f}{4} \frac{\Sigma_{m}^{2}}{\sigma_{0}^{2}}-(1-f)^{2} \leq 0
$$

and constitutes an upper bound for the yield surface of the porous medium.

It is convenient to recall that the Gurson criterion violates this upper bound for low values of the stress triaxiality $T=\Sigma_{m} / \Sigma_{e q}$. This has motivated modifications such as the ones proposed by Tvergaard [31] or [17] and widely used in literature for numerical applications. However, due to the heuristic nature of these modifications, they are not considered in the following comparative analysis.

The results are shown on figure 1 to 3 for three values of porosity, $f=0.01$, $f=0.1$ and $f=0.2$. On these figures are also reported data numerically obtained by computing the exact value of $\Gamma\left(\boldsymbol{D}, \overline{\boldsymbol{d}}^{*}\right)$ given by $(24)$ without any approximation. For this "exact" solution, the limit analysis of the hollow sphere is performed by the consideration of the Eshelby type velocity field. So, one has to compute $\Gamma\left(\boldsymbol{D}, \overline{\boldsymbol{d}}^{*}\right)$ by integrating the local dissipation (24) and to minimize $\Gamma\left(\boldsymbol{D}, \overline{\boldsymbol{d}}^{*}\right)$ according to the unknown kinematical parameters, $\overline{\boldsymbol{d}}^{*}$. In a second time, the macroscopic criterion is obtained from (7). These numerical results validate the approximations made for the derivation of the closed-form criterion (41).

We compare now the new criterion to the one obtained from variational homogenization techniques and to the Gurson one. Note first that for low values 
of porosity (see figure 1), the new criterion seems to coincide with the Gurson one. The comparison also shows that for higher porosities, the established criterion provides a significant modification of the Gurson criterion for low triaxialities. Moreover, it appears that the new criterion is close to the one predicted by the variational technique for low stress triaxialities while it is interestingly in agreement with the Gurson criterion at high triaxialities. The predictions of the different criteria for pure deviatoric macroscopic stress states (triaxiality equal to 0) are illustrated on figure (4) for various values of porosities.

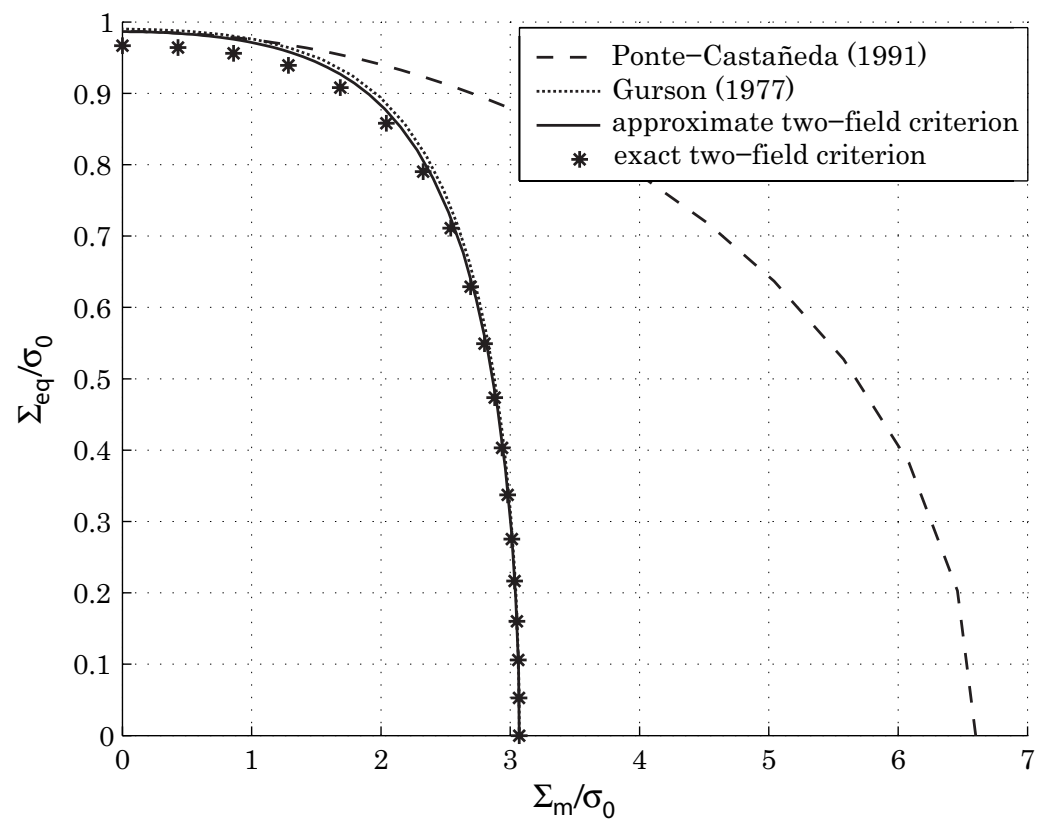

Fig. 1. Comparison between the new criterion (41), Gurson's model (8), the Hashin-Shtrikman bound (43) and the numerical exact solution (obtained by using Eshelby-type velocity fields). The porosity has been chosen equal to $f=0.01$. 


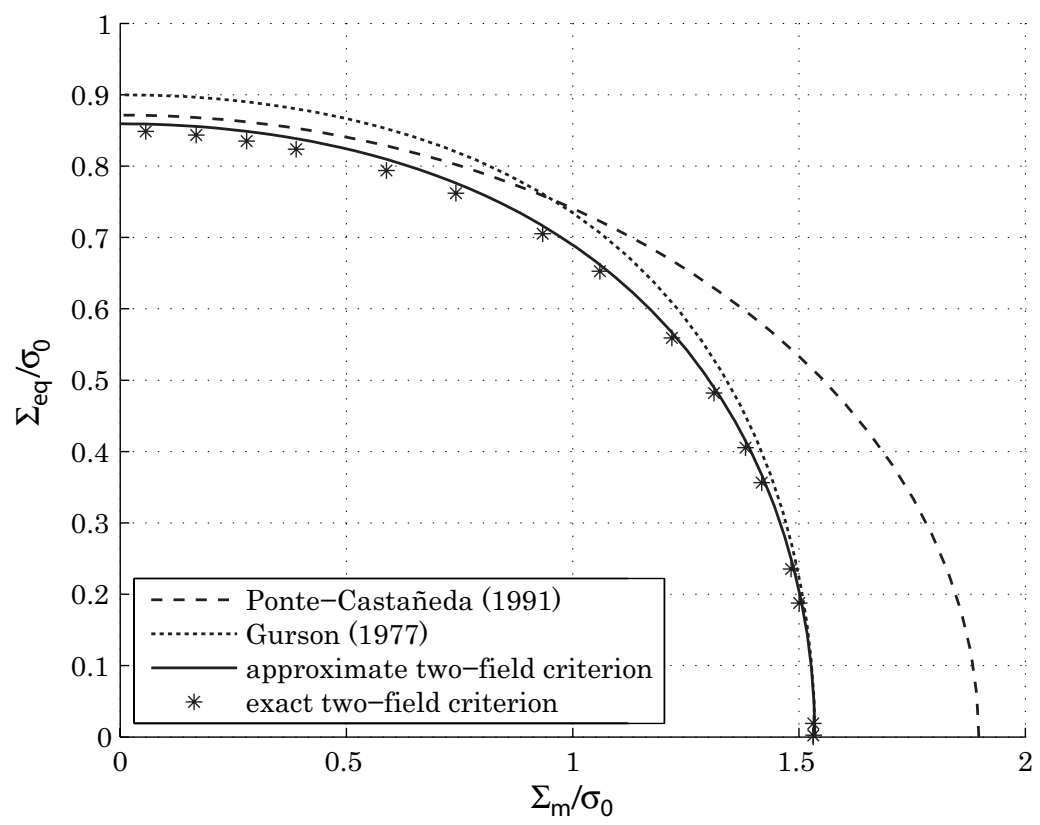

Fig. 2. Comparison between the new criterion (41), Gurson's model (8), the Hashin-Shtrikman bound (43) and the numerical exact solution (obtained by using Eshelby-type velocity fields). The porosity has been chosen equal to $f=0.1$.

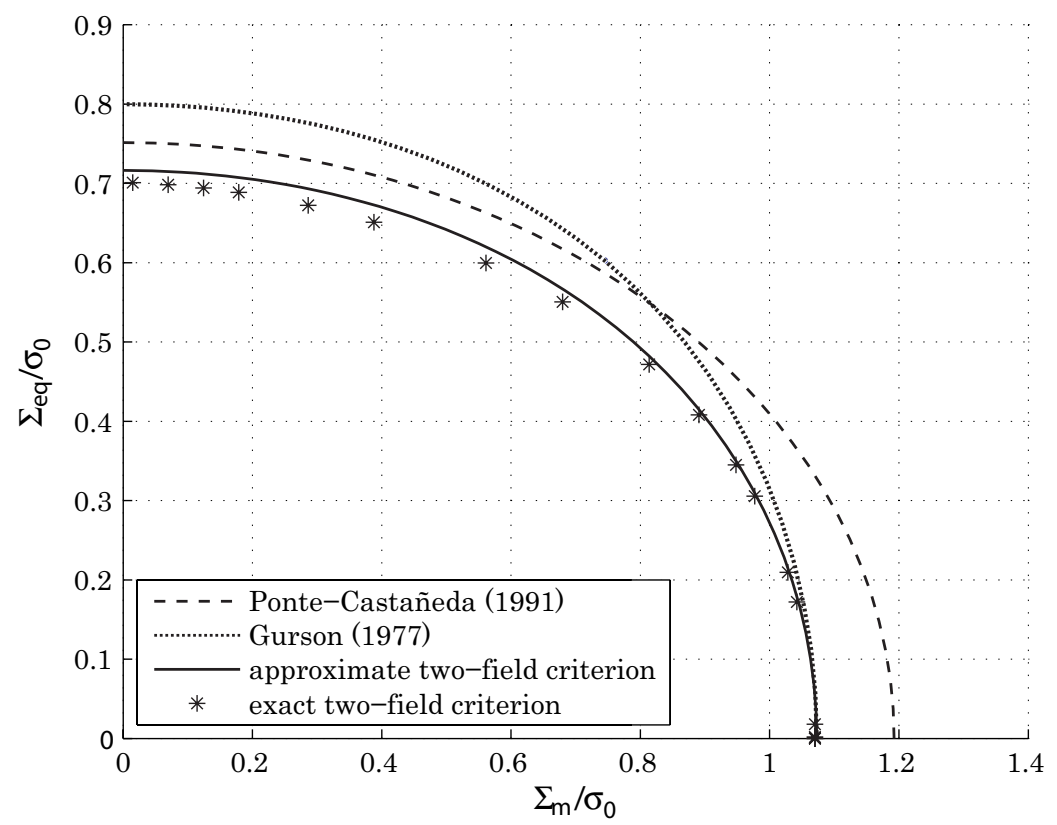

Fig. 3. Comparison between the new criterion (41), Gurson's model (8), the Hashin-Shtrikman bound (43) and the numerical exact solution (obtained by using Eshelby-type velocity fields). The porosity has been chosen equal to $f=0.2$. 


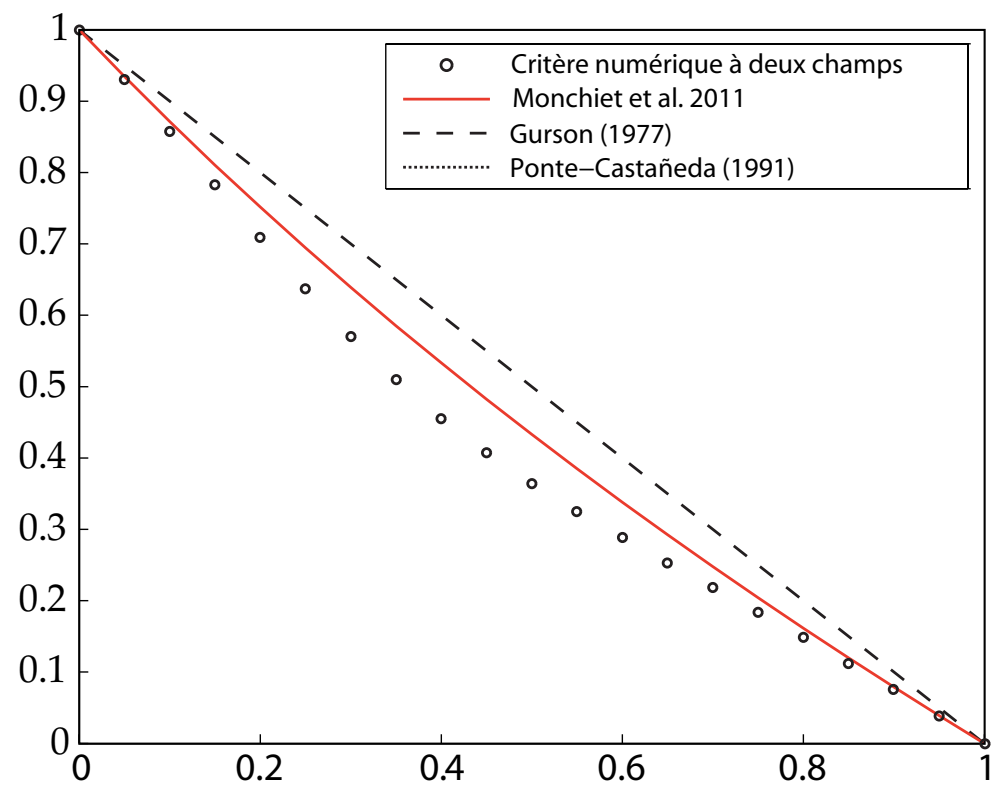

Fig. 4. Value of the equivalent stress as function of the porosity $f$. Comparison between the new criterion (41), Gurson's model (8), the Hashin-Shtrikman bound (43) and the numerical exact solution (obtained by using Eshelby-type velocity fields).

For completeness, it must be emphasized that the very recent works by Danas et al. [2], using a second order method, lead to significant improvements of the predictions provided by non linear homogenization techniques. Note however that the latter do not deliver a closed-form expression of the macroscopic yield function.

\subsection{Comparison to numerical limit analysis results}

As mentioned in section 2.3, boundary conditions used for the derivation of the closed form criterion can be interpreted as homogeneous stress boundary conditions. The determination of the macroscopic criterion has been made by using a kinematical approach and so leads to an upper bound of the macroscopic yield surface for these stress boundary conditions. However to obtain the closed form expression (41), two approximations have been done. The first one, $\mathcal{A} 1$, already used by Gurson, has the advantage to preserve the upper bound character. But, the second approximation $(\mathcal{A} 2)$ is "uncontrolled". In order to assess the accuracy of this approximation, we now propose, in table 1, 2 and 3, some comparisons with numerical solutions recently reported by Trillat and Pastor [30]. These comparisons are made for the following values of the porosity: $f=0.01, f=0.064$ and $f=0.1$. The numerical data given in 
table 1 , corresponding to the porosity $f=0.1$, are reported on figure 5 .

In each table, are given the ratio between the macroscopic equivalent stress and the yield shear stress, namely $\Sigma_{e q} / \tau_{0}$ (with $\sigma_{0}=\tau_{0} \sqrt{3}$ ) for a number of fixed values of the ratio $\Sigma_{m} / \tau_{0}$. The last line in each table provides the numerical value of the ratio $\Sigma_{m}^{\max } / \tau_{0}$ corresponding to $\Sigma_{m} / \tau_{0}$ associated to $\Sigma_{e q}=0$ (purely hydrostatic macroscopic stress).

In the first column, are given the prediction of the new approximate criterion (41) whose accuracy is first compared to the "exact" numerical solution without any approximation. These numerical data are reported in the second column of each table. It can be first observed that, for the three porosities, and whatever the value of the stress triaxiality $T=\Sigma_{m} / \Sigma_{e q}$, the approximated criterion (41) is close to the exact two-field criterion. Moreover, it appears that, despite the approximations required for its derivation, (41) preserves (for the problem with homogeneous stress boundary conditions) the upper bound character of the approach.

To evaluate the accuracy of the above results, we now propose some comparisons with data provided by Trillat and Pastor [30] and numerically obtained by means of Finite Element-based limit analysis of the hollow sphere combined with convex optimization. In [30], the unit cell is subjected either to a homogeneous strain rate conditions $(\underline{v}=\boldsymbol{D} \cdot \underline{x})$ or, as in the present study, to homogeneous stress boundary conditions $(\boldsymbol{\sigma} \cdot \underline{n}=\boldsymbol{\Sigma} . \underline{n})$. For each boundary conditions, the problem is solved by means of a static approach (leading to a lower bound for the macroscopic criterion) and a kinematical approach (giving an upper bound). Tables 1, 2 and 3 give the numerical results corresponding to homogeneous stress boundary conditions (BC) and strain rate boundary conditions. It is generally observed that, comparatively to homogeneous stress boundary conditions, the values obtained in the present study are larger than the upper bound provided by the Finite Element bounds. This can also be clearly observed on figure 5 . 


\begin{tabular}{|c|c|c|c|c|c|c|}
\hline \multirow[t]{2}{*}{$\Sigma_{m} / \sigma_{0}$} & \multicolumn{2}{|c|}{ Present approach } & \multicolumn{2}{|c|}{$\begin{array}{c}\text { Trillat and Pastor's results } \\
\text { stress BC }\end{array}$} & \multicolumn{2}{|c|}{$\begin{array}{c}\text { Trillat and Pastor's results: } \\
\text { strain rate BC }\end{array}$} \\
\hline & $\begin{array}{l}\text { approx. } \\
\text { criterion }\end{array}$ & $\begin{array}{l}\text { exact } \\
2 \quad \text { fields } \\
\text { criterion }\end{array}$ & static & kinematic & static & kinematic \\
\hline 0 & 1.7084 & & 1.6937 & 1.7048 & 1.7 & 1.7086 \\
\hline 1.6 & 1.6867 & 1.6817 & 1.6732 & 1.6835 & 1.6784 & 1.6894 \\
\hline 3.2 & 1.5711 & 1.5631 & 1.5016 & 1.5511 & 1.5435 & 1.5805 \\
\hline 4 & 1.4058 & 1.3893 & 1.2745 & 1.3713 & 1.3452 & 1.4271 \\
\hline 4.4 & 1.2564 & 1.2290 & 1.0705 & 1.2214 & 1.1568 & 1.2888 \\
\hline 4.8 & 1.0141 & 0.9674 & 0.7698 & 1.0009 & 0.845 & 1.067 \\
\hline 5 & 0.8245 & 0.7636 & 0.5472 & 0.8467 & 0.5467 & 0.9013 \\
\hline 5.1 & 0.6954 & 0.6150 & 0.2001 & 0.7509 & 0.3856 & 0.7966 \\
\hline 5.2 & 0.5211 & 0.4358 & - & 0.6355 & - & 0.6698 \\
\hline 5.3 & 0.2055 & 0.1649 & - & 0.4872 & - & 0.5084 \\
\hline$\Sigma_{m}^{\max } / \tau_{0}$ & 5.3176 & 5.3175 & 5.1791 & 5.4385 & 5.1746 & 5.4388 \\
\hline
\end{tabular}

Table 1: numerical values of $\Sigma_{e q} / \tau_{0}$ versus $\Sigma_{m} / \tau_{0}$ corresponding to $f=0.01$ 


\begin{tabular}{|c|c|c|c|c|c|c|}
\hline \multirow[t]{2}{*}{$\Sigma_{m} / \sigma_{0}$} & \multicolumn{2}{|c|}{ Present approach } & \multicolumn{2}{|c|}{$\begin{array}{c}\text { Trillat and Pastor's results } \\
\text { stress BC }\end{array}$} & \multicolumn{2}{|c|}{$\begin{array}{c}\text { Trillat and Pastor's results: } \\
\text { strain rate BC }\end{array}$} \\
\hline & $\begin{array}{l}\text { approx. } \\
\text { criterion }\end{array}$ & $\begin{array}{l}\text { exact } \\
2 \quad \text { fields } \\
\text { criterion }\end{array}$ & static & kinematic & static & kinematic \\
\hline 0 & 1.5776 & 1.5671 & 1.4421 & 1.4963 & 1.5848 & 1.5911 \\
\hline 0.8 & 1.5454 & 1.5253 & 1.4276 & 1.4749 & 1.5589 & 1.5651 \\
\hline 1.6 & 1.4298 & 1.4140 & 1.2836 & 1.3235 & 1.4417 & 1.4532 \\
\hline 2 & 1.3200 & 1.3070 & 1.1661 & 1.1922 & 1.3241 & 1.3420 \\
\hline 2.4 & 1.1483 & 1.1358 & 0.9642 & 1.0106 & 1.1330 & 1.1621 \\
\hline 2.8 & 0.8570 & 0.8369 & 0.6791 & 0.7396 & 0.7942 & 0.8528 \\
\hline 3 & 0.6063 & 0.5747 & 0.3968 & 0.5120 & 0.5057 & 0.5978 \\
\hline 3.1 & 0.4029 & 0.3658 & - & 0.3613 & 0.2618 & 0.4077 \\
\hline$\Sigma_{m}^{\max } / \tau_{0}$ & 3.0701 & 3.0699 & 3.1085 & 3.1911 & 3.1086 & 3.1915 \\
\hline
\end{tabular}

Table 2: numerical values of $\Sigma_{e q} / \tau_{0}$ versus $\Sigma_{m} / \tau_{0}$ corresponding to $f=0.064$ 


\begin{tabular}{|c|c|c|c|c|c|c|}
\hline \multirow[t]{2}{*}{$\Sigma_{m} / \sigma_{0}$} & \multicolumn{2}{|c|}{ Present approach } & \multicolumn{2}{|c|}{$\begin{array}{c}\text { Trillat and Pastor's results } \\
\text { stress BC }\end{array}$} & \multicolumn{2}{|c|}{$\begin{array}{c}\text { Trillat and Pastor's results: } \\
\text { strain rate BC }\end{array}$} \\
\hline & $\begin{array}{l}\text { approx. } \\
\text { criterion }\end{array}$ & $\begin{array}{l}\text { exact } \\
2 \quad \text { fields } \\
\text { criterion }\end{array}$ & static & kinematic & static & kinematic \\
\hline 0 & 1.4882 & 1.4736 & $\begin{array}{l}1.3913 \\
(1.3774)\end{array}$ & $\begin{array}{l}1.4213 \\
(1.4150)\end{array}$ & $\begin{array}{l}1.5020 \\
(1.4805)\end{array}$ & $\begin{array}{l}1.5206 \\
(1.5206)\end{array}$ \\
\hline 0.8 & 1.4368 & 1.4113 & $\begin{array}{l}1.3621 \\
(1.3430)\end{array}$ & $\begin{array}{l}1.3853 \\
(1.3849)\end{array}$ & $\begin{array}{l}1.4598 \\
(1.4624)\end{array}$ & $\begin{array}{l}1.4774 \\
(1.4774)\end{array}$ \\
\hline 1.6 & 1.2473 & 1.2297 & $\begin{array}{l}1.1557 \\
(1.1550)\end{array}$ & $\begin{array}{l}1.1875 \\
(1.1872)\end{array}$ & $\begin{array}{l}1.2699 \\
(1.2669)\end{array}$ & $\begin{array}{l}1.2869 \\
(1.2868)\end{array}$ \\
\hline 2 & 1.0562 & 1.0451 & $\begin{array}{l}0.9490 \\
(0.9436)\end{array}$ & $\begin{array}{l}0.9913 \\
(0.9912)\end{array}$ & $\begin{array}{l}1.0373 \\
(1.0373)\end{array}$ & $\begin{array}{l}1.0820 \\
(1.0820)\end{array}$ \\
\hline 2.2 & 0.9136 & 0.9038 & $\begin{array}{l}0.8039 \\
(0.7918)\end{array}$ & $\begin{array}{l}0.8511 \\
(0.8511)\end{array}$ & $\begin{array}{l}0.8729 \\
(0.8598)\end{array}$ & $\begin{array}{l}0.9269 \\
(0.9269)\end{array}$ \\
\hline 2.4 & 0.7113 & 0.6994 & $\begin{array}{l}0.5789 \\
(0.5973)\end{array}$ & $\begin{array}{l}0.6608 \\
(0.6608)\end{array}$ & $\begin{array}{l}0.6347 \\
(0.6315)\end{array}$ & $\begin{array}{l}0.7108 \\
(0.7106)\end{array}$ \\
\hline 2.5 & 0.5674 & 0.5525 & $\begin{array}{l}0.4504 \\
(0.4318)\end{array}$ & $\begin{array}{l}0.5305 \\
(0.5305)\end{array}$ & $\begin{array}{l}0.4300 \\
(0.4621)\end{array}$ & $\begin{array}{l}0.5632 \\
(0.5601)\end{array}$ \\
\hline$\Sigma_{m}^{\max } / \tau_{0}$ & 2.6587 & 2.6587 & $\begin{array}{l}2.6239 \\
(2.6229)\end{array}$ & $\begin{array}{l}2.6705 \\
(2.6704)\end{array}$ & $\begin{array}{l}2.6316 \\
(2.5650)\end{array}$ & $\begin{array}{l}2.6707 \\
(2.6316)\end{array}$ \\
\hline
\end{tabular}

Table 3: numerical values of $\Sigma_{\text {eq }} / \tau_{0}$ versus $\Sigma_{m} / \tau_{0}$ corresponding to $f=0.1$.

The values within parenthesis correspond to the initial results in [30]

(Numerical results without parenthesis are recent results kindly communicated by the J. Pastor). 


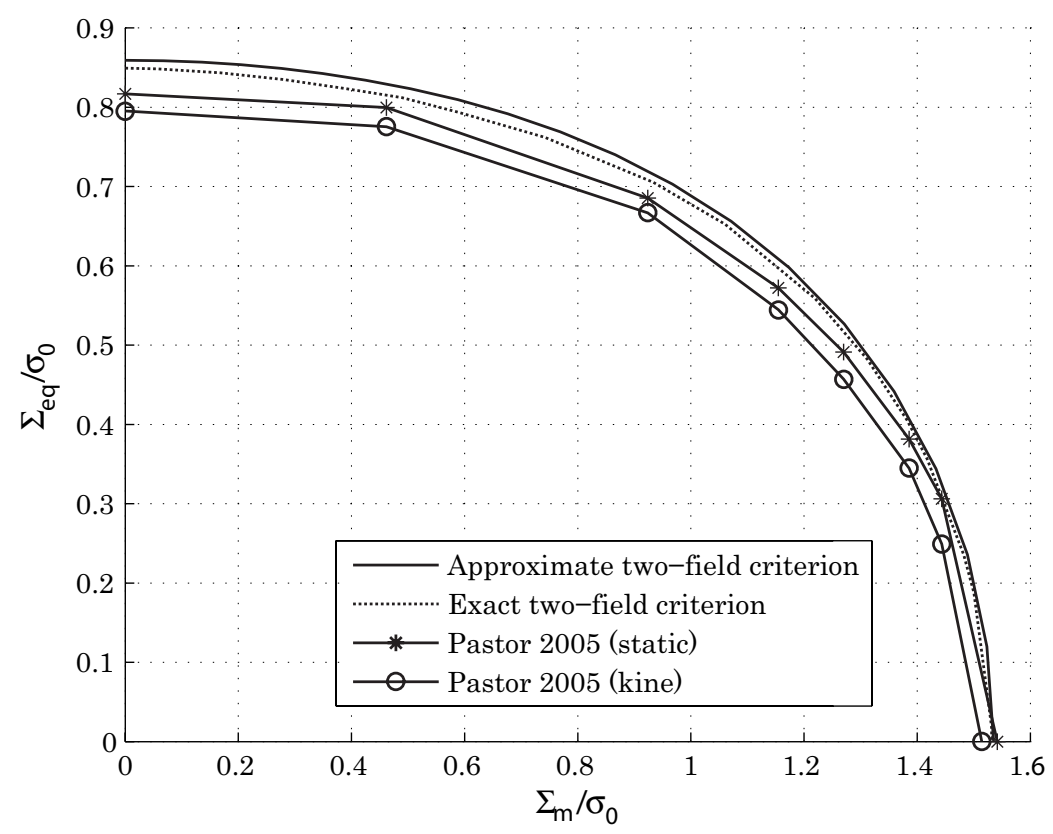

Fig. 5. Comparison between the new criterion (41), numerical results provided by Trillat and Pastor [30], and exact solution, obtained numerically, of the problem depicted in section 3.1 for the porosity $f=0.1$.

For sake of completeness, it can be useful to compare in table 1 to 3 our results to the ones given by the Finite Element bounds in the case of homogeneous strain rate boundary conditions. Interestingly, it appears that our results compare well to these numerical bounds.

\section{Conclusion}

A micromechanics-based modification of the classical Gurson criterion for porous media has been derived. The approach has been developed for a hollow sphere in the framework of limit analysis. The originality of the present study lies in the consideration of new trial velocity fields inspired from the Eshelby exterior point solution to inclusions problem in which the eigenstrains are unknown. Due to the presence of these unknowns, the new estimate of the macroscopic yield criterion has been obtained by implementing a minimization procedure. It has been shown that the derived criterion exhibits some remarkable properties:

- the new criterion is of Gurson type (i.e. non elliptic), but has the significant difference to contain the equivalent stress, $\Sigma_{e q}$, into the hyperbolic cosine while preserving the accuracy of the Gurson model for high stress triaxialities. In particular, as for the Gurson model, the new criterion allows to 
retrieve the exact solution for hydrostatic loadings.

- the most important modification of the Gurson criterion appears for low stress triaxialities, where the derived yield surface is close to the HashinShtrikman bound established by Ponte-castañeda [24] in the framework of the non-linear homogenization applied to a representative elementary volume of isotropic porous media.

In addition to these general remarks, the good accuracy of the various approximations used to obtain the closed-form expression of the yield function is shown by means of comparison with the numerical exact two-field criterion. Moreover, complete evaluation of the criterion is provided by comparisons with recent numerical upper and lower bounds reported by [30]. Both homogeneous strain rate boundary conditions and homogeneous stress boundary conditions are considered in these numerical computations. It is observed that the obtained estimate of the criterion provides yield surfaces close to the numerical results with homogeneous strain boundary conditions. It must be recalled that the Eshelby-based velocity fields do not comply with homogeneous strain rate boundary conditions for the hollow sphere problem. Consequently, the obtained result must be rigorously interpreted in terms of homogeneous stress boundary conditions. A first step of generalization of the above results in order to formulate a constitutive model of isotropic porous media has been proposed in [15] in which the derived model (and its predictions) is compared to the standard Gurson model and the GTN model.

Moreover, a generalization of the present work to anisotropic microstructures has been already introduced in Monchiet et al. [18] by taking into account voids shape effects. Investigation of ductile porous media made up of a von Mises solid matrix and spheroidal (prolate or oblate) cavities is the subject of a forthcoming paper [20].

\section{References}

[1] A.A. Benzerga, J. Besson, A. Pineau.

Anisotropic ductile fracture. Part II: Theory. Acta Mater., 52, 4639-4650, 2004.

[2] K. Danas and M. I. Idiart and P. Ponte-Castañeda.

A homogenization-based constitutive model for isotropic viscoplastic porous media. Int. J. Solid. Struct., 45 : 3392-3409, 2008.

[3] J. D. Eshelby.

The determination of the elastic field of an ellipsoidal inclusion, and related problem. Proc. R. Soc. Lond., A 241 : 376-396, 1957.

[4] J. D. Eshelby.

The elastic field outside an ellipsoidal inclusion. Proc. R. Soc. Lond., A 252 : 561-569, 1959. 
[5] M. Gãrãjeu.

Contribution à l'étude du comportement non linéaire de milieux poreux avec ou sans renfort. PhD Thesis, University of Marseille, 1995.

[6] M. Gãrãjeu, J.C. Michel, P. Suquet.

A micromechanical approach of damage in viscoplastic materials by evolution in size, shape and distribution of voids. Comput. Methods Appl. Mech. Engrg., $183: 223-246,2000$.

[7] M. Gologanu, J.-B. Leblond, J. Devaux.

Approximate models for ductile metals containing non-spherical voids - case of axisymmetric prolate ellipsoidal cavities. J. Mech. Phys. Solids, 41 (11), 1723 Ü1754, 1993.

[8] M. Gologanu, J.B. Leblond, G. Perrin, J. Devaux.

Approximate models for ductile metals containing non-spherical voids - case of axisymmetric oblate ellipsoidal cavities. J. Engrg. Mat. Tech., 116 : 290-297, 1994.

[9] M. Gologanu, J.B. Leblond, G. Perrin and J. Devaux.

Recent extensions of Gurson's model for porous ductile metals. Continuum Micromechanics, ed. P. Suquet, Springer Verlag, 1997.

[10] A.L. Gurson.

Continuum theory of ductile rupture by void nucleation and growth: Part I. Yield criterion and flow rules for porous ductile media. J. Eng. Mat. Tech. 99 : 2-15, 1977.

[11] M. Kachanov, B. Shafiro, I. Tsukrov.

Hanbook of elasticity solutions. Kluwer academic publisher, 2003.

[12] J.-B. Leblond.

Mécanique de la rupture fragile et ductile. Hermes, 2003.

[13] J.B. Leblond, G. Perrin, P. Suquet.

Exact results and approximate models for porous viscoplastic solids. Int. J. Plasticity, 10, 213-235, 1994.

[14] J. Lin, T. Kanit, V. Monchiet, J.-F. Shao, D. Kondo.

Numerical implementation of a recent improved Gurson-type model and application to ductile fracture. Computational Materials Science, 47, 901-906, 2010 .

[15] F.A. McClintock.

A criterion for ductile fracture by the growth of holes. ASME J. Appl. Mech. $35: 363-371,1968$.

[16] J.C. Michel and P. Suquet.

The constitutive law of nonlinear viscous and porous materials. J. Mech. Phys. Solids, 40 : 783-812, 1992. 
[17] V. Monchiet and E. Charkaluk and D. Kondo.

An improvement of Gurson-type models of porous materials by using Eshelbylike trial velocity fields. C.R. Mécanique, 335: 32-41, 2007.

[18] V. Monchiet, O. Cazacu, E. Charkaluk, D. Kondo.

Macroscopic yield criteria for plastic anisotropic materials containing spheroidal voids. Int. J. Plasticity, 24 : 1158-1189, 2008.

[19] V. Monchiet, E. Charkaluk, D. Kondo.

Macroscopic criteria for ductile materials containing spheroidal voids: an Eshelby-like velocity fields approach. In preparation.

[20] T. Mura.

Micromechanics of Defects in Solids. Martinus Nijhoff, Dordrecht. 1987.

[21] Pardoen, T., Hutchinson, J.W..

An extended model for void growth and coalescence. J. Mech. Phys. Solids, 48, 2467-2512, 2000.

[22] P. Ponte-Castañeda.

The effective mechanical properties of nonlinear isotropic composites. J. Mech. Phys. Solids, 39 : 45-71, 1991.

[23] P. Ponte-Castañeda, M. Zaidman.

Constitutive models for porous materials with evolving microstructure. J. Mech. Phys. Solids, 42 : 1459-1492, 1994.

[24] J. R. Rice and D. M. Tracey.

On a ductile enlargement of voids in triaxial stress fields. J. Mech. Phys. Solids, 17 : 201-217, 1969.

[25] P. Suquet.

On bounds for the overall potential of power law materials containing voids with an arbitrary shape. Mech. Res. Com., 19 : 51-58, 1992.

[26] M. Trillat, J. Pastor.

Limit analysis and Gurson's model. Eur. J. Mech. A/Solids, 24 : 800-819, 2005.

[27] V. Tvergaard.

Influence of voids on shear band instabilities under plane strain conditions. Int. J. Frac., 17 : 89-407, 1981.

[28] V. Tvergaard, A. Needleman.

Analysis of cup-cone fracture in round tensile bar. Acta. Metall., 32 : 157-169, 1984.

\section{A Expression of $\bar{d}^{*}$}

From relations (36) with (37) and (38), one has: 


$$
\Sigma_{e q} \frac{\boldsymbol{A}}{A_{e q}}=\frac{3 g(f)}{5} \frac{\Sigma_{b}}{B} \overline{\boldsymbol{d}}^{*}
$$

which can also be put on the form:

$$
\frac{\boldsymbol{A}}{A_{e q}}=\frac{\overline{\boldsymbol{d}}^{*}}{d_{e q}^{*}}
$$

where $d_{e q}^{*}$ is given by:

$$
\frac{d_{e q}^{*}}{B}=\frac{5 B}{3 g(f)} \frac{\Sigma_{e q}}{\Sigma_{b}}
$$

In the last relation $B$ is also function of $d_{e q}^{*}$ (see equation (33)), the computation of $d_{e q}^{*}$ gives:

$$
d_{e q}^{*}=\frac{20}{9} \frac{\Sigma_{e q}}{\Sigma_{m}} D_{m}
$$

Let us recall that $\overline{\boldsymbol{D}}=\boldsymbol{A}+\frac{2 f}{5} \overline{\boldsymbol{d}}^{*}$, so from (A.2) it appears that the directions of $\boldsymbol{A}, \overline{\boldsymbol{d}}^{*}$ and $\overline{\boldsymbol{D}}$ are the same:

$$
\frac{\boldsymbol{A}}{A_{e q}}=\frac{\overline{\boldsymbol{d}}^{*}}{d_{e q}^{*}}=\frac{\overline{\boldsymbol{D}}}{D_{e q}}
$$

Finally, $\overline{\boldsymbol{d}}^{*}$ can read:

$$
\overline{\boldsymbol{d}}^{*}=\frac{20}{9} \frac{\Sigma_{e q}}{D_{e q}} \frac{D_{m}}{\Sigma_{m}} \overline{\boldsymbol{D}}
$$

\section{B Alternative expression of the macroscopic yield function}

We aim at presenting here an alternative form of the macroscopic yield function of the porous medium. To this end, instead of approximation $\mathcal{A} 2$ (see section 3.1$)$, we use in (30) giving $\Gamma(\boldsymbol{D}, \overline{\boldsymbol{d}})$ the following alternative one:

$\mathcal{A} 2^{*}$ : function $6 G(u) u^{2} / 25$ is replaced by a constant denoted $h(f)$.

$\Gamma(\boldsymbol{D}, \overline{\boldsymbol{d}})$ is then approximated by: 


$$
\Gamma(\boldsymbol{D}, \overline{\boldsymbol{d}})=\sigma_{0} f \int_{u=f}^{u=1} \sqrt{A_{e q}^{2}+h(f) d_{e q}^{* 2}+4 d_{m}^{* 2} u^{2}} \frac{d u}{u^{2}}
$$

The approximation consisting to replace $6 G(u) u^{2} / 25$ by its average value over the interval $[u 1, u 2]=[f, 1]$ appears to be poor here. A better approximation has be found by introducing the change of variable $w=f / u$ in (B.1) :

$$
\Gamma(\boldsymbol{D}, \overline{\boldsymbol{d}})=\sigma_{0} \int_{w=f}^{w=1} \sqrt{A_{e q}^{2}+H(w) d_{e q}^{* 2}+\frac{4 D_{m}^{2}}{w^{2}}} d w
$$

with:

$$
H(w)=\frac{2}{25}\left(15-40(f / w)^{2 / 3}+28(f / w)^{4 / 3}\right)(f / w)^{2}
$$

and by replacing $H(w)$ by its average over the interval $[w 1, w 2]=[f, 1]$. This leads to the following expression for $h(f)$ :

$$
h(f)=\frac{1}{1-f} \int_{f}^{1} H(w) d w=\frac{6 f g(f)}{25}
$$

where $g(f)$ is defined by (31). Now, the integration of (B.2) yields:

$$
\begin{aligned}
\Gamma\left(\boldsymbol{D}, \overline{\boldsymbol{d}}^{*}\right)= & \sigma_{0} \int_{f}^{1} \sqrt{A^{2}+\frac{4 D_{m}^{2}}{w^{2}}} d w \\
& =\sigma_{0}\left[B \operatorname{arcsinh}\left(\frac{2 D_{m}}{A w}\right)-\sqrt{A^{2} w^{2}+B^{2}}\right]_{f}^{1}
\end{aligned}
$$

with:

$$
A=\sqrt{A_{e q}^{2}+h(f)\left(d_{e q}^{*}\right)^{2}}
$$

At this stage, it is interesting to notice that the macroscopic dissipation is exactly the one derived by Gurson, $A_{e q}$ being replaced here by $A$ whose expression has to be established now.

As $\Gamma\left(\boldsymbol{D}, \overline{\boldsymbol{d}}^{*}\right)$ only depends on $\overline{\boldsymbol{d}}^{*}$ through the scalar $A$, the minimum of $\Gamma\left(\boldsymbol{D}, \overline{\boldsymbol{d}}^{*}\right)$ according to $\overline{\boldsymbol{d}}^{*}$ is obtained for:

$$
\frac{\partial A}{\partial \overline{\boldsymbol{d}}^{*}}=0
$$

Since $\boldsymbol{A}=\overline{\boldsymbol{D}}-\frac{2 f}{5} \overline{\boldsymbol{d}}^{*}$ (see equation (22)), it can be shown that the minimum is attained for: 


$$
\overline{\boldsymbol{d}}^{*}=\frac{\frac{2 f}{5}}{\frac{4 f^{2}}{25}+h(f)} \overline{\boldsymbol{D}}
$$

which appears to be more simple than (A.6).

It follows that $A$ is given by:

$$
A^{2}=\frac{h(f)}{\frac{4 f^{2}}{25}+h(f)} D_{e q}^{2}=\frac{g(f)}{\frac{2 f}{3}+g(f)} D_{e q}^{2}
$$

Putting (B.9) in (B.5) yields the macroscopic dissipation $\Pi(\boldsymbol{D})$. Recalling that the macroscopic dissipation is the one derived by Gurson with $A_{e q}$ replaced by $A$, the macroscopic criterion is obtained by a change of variable:

$$
\left(1+\frac{2}{3} \frac{f}{g(f)}\right) \frac{\Sigma_{e q}^{2}}{\sigma_{0}^{2}}+2 f \cosh \left(\frac{3}{2} \frac{\Sigma_{m}}{\sigma_{0}}\right)-1-f^{2} \leq 0
$$

It is worth noticing that, for low porosities $(f \simeq 0)$ :

$$
1+\frac{2}{3} \frac{f}{g(f)} \simeq 1+\frac{2}{3} f+o\left(f^{2}\right)
$$

which leads to the following approximate expression of the macroscopic yield function for low values of porosities:

$$
\left(1+\frac{2}{3} f\right) \frac{\Sigma_{e q}^{2}}{\sigma_{0}^{2}}+2 f \cosh \left(\frac{3}{2} \frac{\Sigma_{m}}{\sigma_{0}}\right)-1-f^{2} \leq 0
$$

Remarkably, this expression is exactly the one heuristically proposed by [14]. It must be emphasized that the alternative criterion (B.10), still preserve the exact solution for purely hydrostatic loading, i.e $\Sigma_{m}=-\frac{2 \sigma_{0}}{3} \ln (f)$. In the case of purely deviatoric macroscopic stress states $\left(\Sigma_{m}=0\right)$, we represent on figure B.1 the value of the equivalent stress as function of the porosity for the two approximate criterion (41) and (B.10) and the numerical two-field criterion. It can be observed that the predictions of the two approximate criteria (41) and (B.10) are equivalent. 


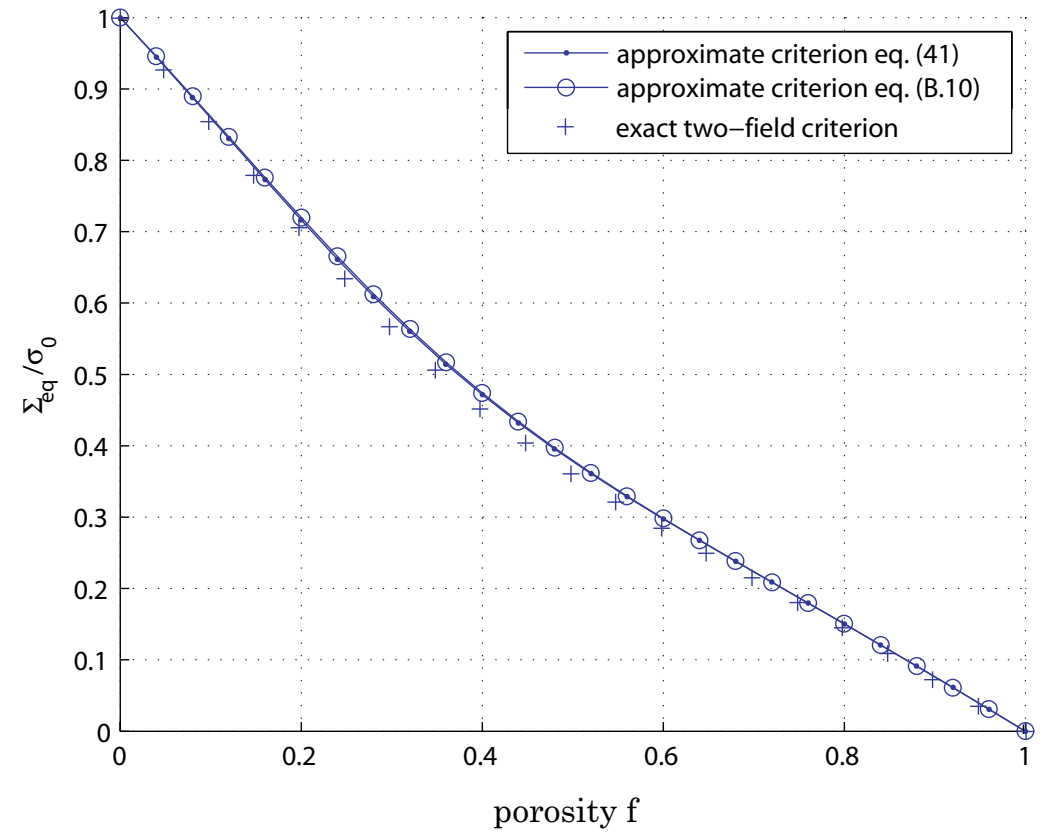

Fig. B.1. Comparison between the approximate criteria (41), (B.10) and numerical data for the exact two field criterion. Value of the equivalent stress as function of the porosity.

Also for comparison purpose, the predictions of the alternative criterion, (B.10), and the previously proposed one, (42), are plotted on figure B.2 for a porosity $f=0.1$. On this figure are also reported the data numerically obtained for the exact two-field solution. Despite the high proximity of the results, it can be observed that the first approximate form (42) is slightly more close to the numerical points than the second form (B.2). expression (42) of the criterion is then preferred. 


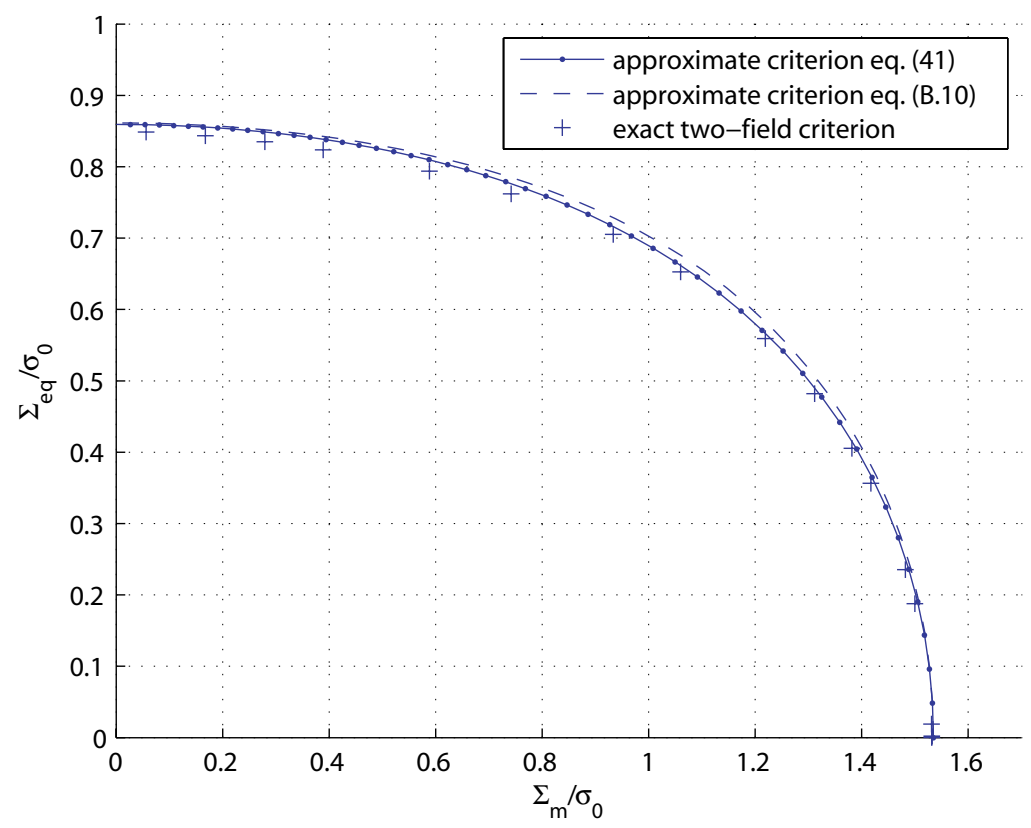

Fig. B.2. Comparison between the approximate criteria (41), (B.10) and numerical data for the exact two field criterion. The porosity has been chosen equal to $f=0.1$. 